Invest. Pens. Crit.(ISSN 1812-3864; eISSN 2644-4119)

Vol. 10, No. 1, Enero - Abril 2022. pp. $04-11$

DOI: https://doi.org/10.37387/ipc.v10i1.271

Artículo Científico

\title{
Vínculos parentales tempranos y dependencia emocional en las relaciones de pareja de jóvenes universitarios panameños
}

\section{Early parental bonds and emotional dependency in couple relationships of young Panamanian university students}

\author{
Jenifer Pinzón Agrazal ${ }^{1, *}$ \& Orquídea Robles De Polo, ${ }^{2, *}$ \\ ${ }^{1}$ Facultad de Psicología, Escuela de Psicología, Universidad de Panamá \\ Dhttps://orcid.org/0000-0002-1906-5651
}

${ }^{2}$ Facultad de Psicología, Escuela de Psicología, Universidad de Panamá

https://orcid.org/0000-0001-9710-468X

* Autor por correspondencia: Jenifer Pinzón Agrazal, jenpinzon4@gmail.com

Orquídea Robles De Polo, orquidearoblesdepolo@gmail.com

Recibido: 09 de noviembre de 2021

Aceptado: 12 de diciembre de 2021

\section{Resumen}

Introducción: El vínculo parental nos habla de la percepción que se tiene sobre la conducta y actitud de uno o ambos padres en relación con aspectos de la infancia o adolescencia del sujeto. Este concepto está basado en la teoría del apego desarrollada por el psicoanalista inglés John Bowlby, el cual describe los efectos que producen las experiencias tempranas y la relación con la primera figura vincular en el desarrollo del niño. Objetivo: El objetivo de esta investigación fue determinar la relación que existe entre los vínculos parentales tempranos y la dependencia emocional en las relaciones de pareja de jóvenes universitarios panameños. Materiales y método: Se realizó un estudio de diseño no experimental de tipo transeccional y relacional, fue aplicado a una muestra de 383 estudiantes universitarios de la República de Panamá. Para la recolección de datos se utilizó el Instrumento de Vínculo Parental (Parental Bonding Instrument, PBI) de Parker, Tupling and Brown (1979) y el Inventario de Dependencia Emocional (IDE) creado por el psicólogo Jesús Joel Aiquipa Tello (2012). Los datos se analizaron de manera descriptiva y con el estadístico chicuadrado. Resultados: Aproximadamente la mitad de los participantes mostraron un nivel de dependencia emocional que reflejaba una vinculación afectiva patológica y no adaptativa en sus relaciones de pareja. Un porcentaje significativo de estos participantes también reflejó un apego inseguro hacia la figura paterna. Discusión: Los resultados encontrados coinciden con lo planteado por la literatura, que indica que las características, positivas o negativas del estilo de apego con nuestros padres o cuidadores en la infancia, van a determinar la calidad de nuestras relaciones afectivas en la vida adulta. Conclusiones: Existe una relación estadísticamente significativa entre el nivel de dependencia emocional y el tipo de apego hacia los padres o cuidadores.

Palabras clave: dependencia emocional; vínculo parental; apego; pareja; jóvenes. 


\begin{abstract}
Introduction: the parental bond talks about the perception of the behavior and attitude of one or both parents concerning the subject's childhood or adolescence. The concept is based on attachment theory, developed by the English psychoanalyst John Bowlby, which describes the effects produced by early experiences and the relationship with the first bonding figure in the child's development. Objective: The objective of this research was to determine the link between early parental bonds and emotional dependency in couple relationships of young Panamanian university students. Material and methods: A non-experimental design study of a cross-sectional and relational type was carried out; it was applied to a sample of 383 university students from the Republic of Panama. For data collection, the Parental Bonding Instrument (PBI) by Parker, Tupling, and Brown (1979) and the Emotional Dependency Inventory (Inventario de Dependencia Emocional, IDE) by the psychologist Jesús Joel Aiquipa Tello (2012) were used. The data were analyzed descriptively and with the chi-square test. Results: Approximately half of the participants showed levels of emotional dependency that reflected pathological and non-adaptive affective bonding in their couple relationships. A significant percentage of those participants also displayed insecure attachment to their father figure. Discussion: The results coincide with what is stated in the literature, which indicates that the negative or positive characteristics of the attachment style with our parents o caretakers during childhood are going to determine the quality of our affective relationships in our adult lives. Conclusion: There exists a statistically significant link between the level of emotional dependency and the attachment style towards the parents or caretakers.
\end{abstract}

Keywords: emotional dependency; parental bond; attachment; couple; young.

\title{
Introducción
}

El vínculo parental nos habla de la percepción que se tiene sobre la conducta y actitud de uno o ambos padres en relación con aspectos de la infancia o adolescencia del sujeto (Estrada, Barneveld, Maya, 2016). Este concepto está basado en la teoría del apego desarrollada por el psicoanalista inglés John Bowlby, el cual describe los efectos que producen las experiencias tempranas y la relación con la primera figura vincular en el desarrollo del niño (Moneta, 2014)

Para respaldar su teoría del apego, Bowlby realizó variadas investigaciones. En 1944 Estudió a 44 jóvenes que habían sido detenidos por robo, al revisar sus biografías encontró evidencias de que, durante la infancia, los mismos habían sido víctimas de abuso y maltratos por parte de sus padres. Bowlby presentó sus hallazgos en un artículo que denominó "cuarenta y cuatro jóvenes ladrones". Además, estudió el efecto que tuvo en niños pequeños, el haber sido separados de sus madres por un periodo significativo de tiempo (Moneta, 2014)

Después de la Segunda Guerra Mundial Bowlby se hace responsable del Departamento de Niños de la Clínica Tavistock, es aquí donde conoce a Mary Ainsworth, una psicóloga canadiense, que empezó a trabajar como investigadora bajo la dirección de Bowlby y posteriormente se convierten en compañeros de investigación. (Carillo, 1999)

Alrededor de 1960, su compañera de investigación Ainsworth diseña una investigación experimental, llamada la situación del extraño, con el objetivo de establecer la relación entre las conductas de apego y la exploración. Confirmando así, que el niño utiliza a la madre como una base segura para explorar, y cuando percibe alguna amenaza o "Situación Extraña" las conductas de apego son activadas, haciendo desaparecer la conducta de exploración. (Delgado, 2004). La metodología de Mary Ainsworth probó empíricamente las ideas de Bowlby y ayudó a expandirlas (Bretherton, 1992).

Las carencias afectivas tempranas y la falta de vinculación con personas significativas impactan, desde los primeros años de vida, en la vida del sujeto (Blasco, 2005). De igual manera, si los padres no le permiten al niño desarrollar capacidades y comportamientos independientes y autónomos, evitan que este construya su ser y su personalidad por sí mismo. Cuando esto pasa el individuo tiene una alta probabilidad de desarrollar en el futuro, una dependencia emocional hacia las personas que lo rodean. (Rodríguez, 2013).

En 2005, Castelló establece que, "la dependencia emocional es la necesidad extrema de carácter afectivo que una persona siente hacia su pareja a lo largo de sus diferentes relaciones".

Siguiendo a este autor, el dependiente emocional presenta un comportamiento sumiso y obsesivo en torno a su pareja, la idealiza y presenta temores de separación. 
La dependencia emocional es reconocida por la investigación realizada por González y Zavala (2013) en el Centro de Apoyo a la Mujer Maltratada (CAMM) como un factor de riesgo para la violencia en la pareja.

Tomando en cuenta que, Panamá es un país con una alta tasa de violencia hacia la mujer, según estadísticas brindadas por el Ministerio Público, el desarrollo de una dependencia emocional es un problema de índole social.

De ahí surge la necesidad de realizar esta investigación. Los resultados servirán para recalcar la importancia del establecimiento de vínculos afectivos fuertes y sanos durante la infancia; que los padres reconozcan que conectarse emocionalmente con los niños, cultivar una buena autoestima y promover la autonomía actúan como factores protectores para prevenir la dependencia emocional, que puede desencadenar no sólo en violencia hacia o por parte de la pareja; sino en conductas antisociales, depresión, ansiedad u otros patrones de comportamiento desadaptativos. Esta investigación tiene como objetivo determinar la relación que existe entre los vínculos parentales tempranos y la dependencia emocional en las relaciones de pareja de jóvenes universitarios panameños.

\section{Método}

\section{Objetivos}

El objetivo de esta investigación fue determinar la relación que existe entre los vínculos parentales tempranos y la dependencia emocional en las relaciones de pareja de jóvenes universitarios panameños.

\section{Población y Muestra}

La población incluyó el total de estudiantes de todas las universidades de la República de Panamá, que según los datos de la dirección de estadística y censo alcanzaban un total de 155,698 estudiantes. Se calculó la muestra con la fórmula para poblaciones finitas. Para esto se utilizó el programa Epi Info 7 y una confianza del $95 \%$.

El estudio tuvo una muestra de 383 estudiantes universitarios de la República de Panamá, entre las edades de 18 a 25 años.

La técnica de muestreo fue no probabilística por conveniencia. Los criterios de inclusión fueron ser un joven panameño entre 18 a 25 años, cursar estudios universitarios en la República de Panamá, haber tenido o tener relaciones de noviazgo, aceptar el consentimiento informado y llenar de forma voluntaria el instrumento.

\section{Instrumento}

Para medir la variable de vínculos parentales tempranos se utilizó el Instrumento de Vínculo Parental (Parental Bonding Instrument, PBI) de Parker, Tupling and Brown (1979). Este instrumento consta de dos escalas: 1. Cuidado (12 ítems) que se refiere, por un lado, a la afectuosidad, contención emocional, empatía y cercanía, y, por otro, la frialdad emotiva, indiferencia, negligencia, y 2. Sobreprotección (13 ítems) que apunta al control, sobreprotección, intrusión, contacto excesivo, infantilización y prevención de la conducta autónoma.

La obtención de puntajes de ambas escalas permitió elaborar cinco tipos de vínculos parentales: control sin afecto (bajo cuidado y alta sobreprotección), constricción afectiva (alto cuidado y alta sobreprotección), vínculo ausente (bajo cuidado y baja sobreprotección), vínculo óptimo (alto cuidado y baja sobreprotección) y vínculo promedio (promedios en ambas escalas).

Sin embargo, el Parental Bonding Instrument (PBI) no contaba con una categoría para aquellos participantes que obtuvieron puntuaciones promedio en sólo una de las escalas (sea sobreprotección o cuidado).

Tomando en cuenta que, aproximadamente la mitad de la muestra cayó en esta categoría no reconocida por el PBI se utilizó la recategorización propuesta por Escobar, M (2008), en el que se brinda un esquema reestructurado, el cual incluye las 5 categorías principales del PBI y se toma en cuenta las nuevas combinaciones que resultan al obtener una puntuación promedio en sólo una de las escalas. Dejándonos con dos categorías globales: Apego seguro y Apego inseguro (Dávila, Ormeño, Vera, 1998-2001).

Para medir la variable de dependencia emocional se utilizó el Inventario de Dependencia Emocional (IDE) creado por el psicólogo Jesús Joel Aiquipa Tello (2012). El inventario de dependencia emocional - IDE, está conformada por 49 ítems agrupados en 7 factores: 1) Miedo a la ruptura, 2) Miedo e intolerancia a la soledad, 3) Prioridad de la pareja, 4) Necesidad de acceso a la pareja, 5) Deseos de exclusividad, 6) Subordinación y sumisión, 7) Deseos de control y dominio. 
La puntuación obtenida en este inventario representó el nivel de dependencia emocional obtenido por el participante: alto (71-99), moderado (51-70), significativo (31-50), bajo o normal (1-30).

\section{Procedimiento de recogida y análisis de datos}

Se trató de un diseño no experimental de tipo transeccional y relacional. La investigación fue divulgada a través de redes sociales, asociaciones estudiantiles, coordinadores de grupos y docentes de las distintas universidades de la República de Panamá con un post informativo en el que se explicó el objetivo de la investigación y los requisitos para participar. Las personas que cumplieron con los requisitos y desearon participar, de forma voluntaria pudieron acceder a un link donde aceptaron el consentimiento informado y respondieron a los dos instrumentos en línea.

Se hizo un análisis descriptivo de las variables vínculos parentales tempranos y dependencia emocional, así como de las características sociodemográficas y personales de los participantes en el estudio. Además, se utilizaron las medidas de tendencia central para el análisis de la variable cuantitativa edad.

Para medir la asociación entre los vínculos parentales tempranos de la figura materna y paterna respectivamente, y la dependencia emocional se utilizó el estadístico chi-cuadrado, ya que, ambas variables daban como resultado categorías. Para el análisis de los datos se utilizó el software SPSS v.23. La investigación fue aprobada y avalada por el Comité de Bioética de la Universidad de Panamá.

\section{Resultados}

Se obtuvo una tasa de respuesta del $80 \%$ (306 participantes)

En la tabla 1 se observa un predominio del del sexo femenino (80.7\%). La edad mínima en cumplimiento de los criterios de inclusión al estudio fue de 18 años y la máxima de 25 años, el mayor porcentaje de participantes tenía 23años (22.2\%) y el menor porcentaje corresponde a los 25 años (4.9\%). Se observa poca dispersión en cuanto a la variable edad con una media de 22 años y una DE 1.95.

Tabla 1. Datos sociodemográficos de los estudiantes universitarios panameños

$\begin{array}{ccc} & \text { DATOS GENERALES } & \\ \text { EDAD } & \mathbf{N} & \% \\ 18 \text { años } & 23 & 7.5 \\ 19 \text { años } & 31 & 10.1 \\ 20 \text { años } & 44 & 14.4 \\ 21 \text { años } & 47 & 15.4 \\ 22 \text { años } & 40 & 13.1 \\ 23 \text { años } & 68 & 22.2 \\ 24 \text { años } & 38 & 12.4 \\ 25 \text { años } & 15 & 4.9 \\ \text { Total } & \mathbf{3 0 6} & \mathbf{1 0 0 . 0} \\ & & \\ \text { SEXO } & \mathbf{N} & \mathbf{\%} \\ \text { Femenino } & 247 & 80.7 \\ \text { Masculino } & 59 & 19.3 \\ \text { Total } & \mathbf{3 0 6} & \mathbf{1 0 0 . 0}\end{array}$

En la tabla 2 se observa que, el 51.6\% de los participantes pertenecían a la Universidad de Panamá, el 18.3\% a la Universidad Tecnológica de Panamá, el 2.9\% a la Universidad de las Américas y el 1.3\% a la Universidad Autónoma de Chiriquí; conformando un $74.1 \%$ perteneciente a instituciones estatales.

El $25.7 \%$ restante pertenecía a entidades privadas como la Universidad Latina de Panamá, la Universidad Santa María La Antigua y otras universidades privadas.

Tabla 2. Institución académica a la que pertenecen los estudiantes universitarios panameños 


\begin{tabular}{ccc}
\hline UNIVERSIDAD & $\mathbf{N}$ & $\mathbf{\%}$ \\
Universidad de Panamá (UP) & 158 & 51.6 \\
Universidad Tecnológica de Panamá (UTP) & 56 & 18.3 \\
Universidad Latina de Panamá & 24 & 7.8 \\
Universidad Santa María La Antigua & 26 & 8.5 \\
(USMA) & & \\
Universidad Interamericana de Panamá & 16 & 5.2 \\
(UIP) & & \\
Universidad de las Américas (UDELAS) & 9 & 2.9 \\
Universidad Autónoma de Chiriquí & 4 & 1.3 \\
Otras universidades privadas & 13 & 4.2 \\
Total & $\mathbf{3 0 6}$ & $\mathbf{1 0 0 . 0}$ \\
\hline
\end{tabular}

La tabla 3 indica que, en el 55.6\% de los participantes, se reflejó un nivel de dependencia emocional bajo o normal, mientras que, el 9.8\% indicó un nivel de dependencia emocional alto.

Tabla 3. Nivel de dependencia emocional

\begin{tabular}{ccc}
\hline NIVEL & N & $\%$ \\
Bajo o normal & 170 & 55.6 \\
Significativo & 74 & 24.2 \\
Moderado & 32 & 10.5 \\
Alto & 30 & 9.8 \\
Total & 306 & 100.0 \\
\hline
\end{tabular}

La figura 1 indica que, el 55.6\% de la muestra con la categoría bajo o normal refleja una vinculación afectiva funcional. Por otra parte, el $44.5 \%$ reveló una vinculación afectiva patológica hacia sus relaciones de pareja. Nota. El Inventario de Dependencia Emocional (IDE) creado por el psicólogo Jesús Joel Aiquipa Tello (2012) mide la dependencia emocional en su dimensión patológica. Se debe recalcar que la categoría "bajo o normal" corresponde a un nivel de dependencia emocional normal o esperado, en donde la vinculación afectiva está presente en niveles funcionales. Sin embargo, en las categorías "Significativo", "Moderado" y "Alto", esta vinculación afectiva es considerada patológica o disfuncional.

Figura 1. Dimensión funcional y patológica de la dependencia emocional

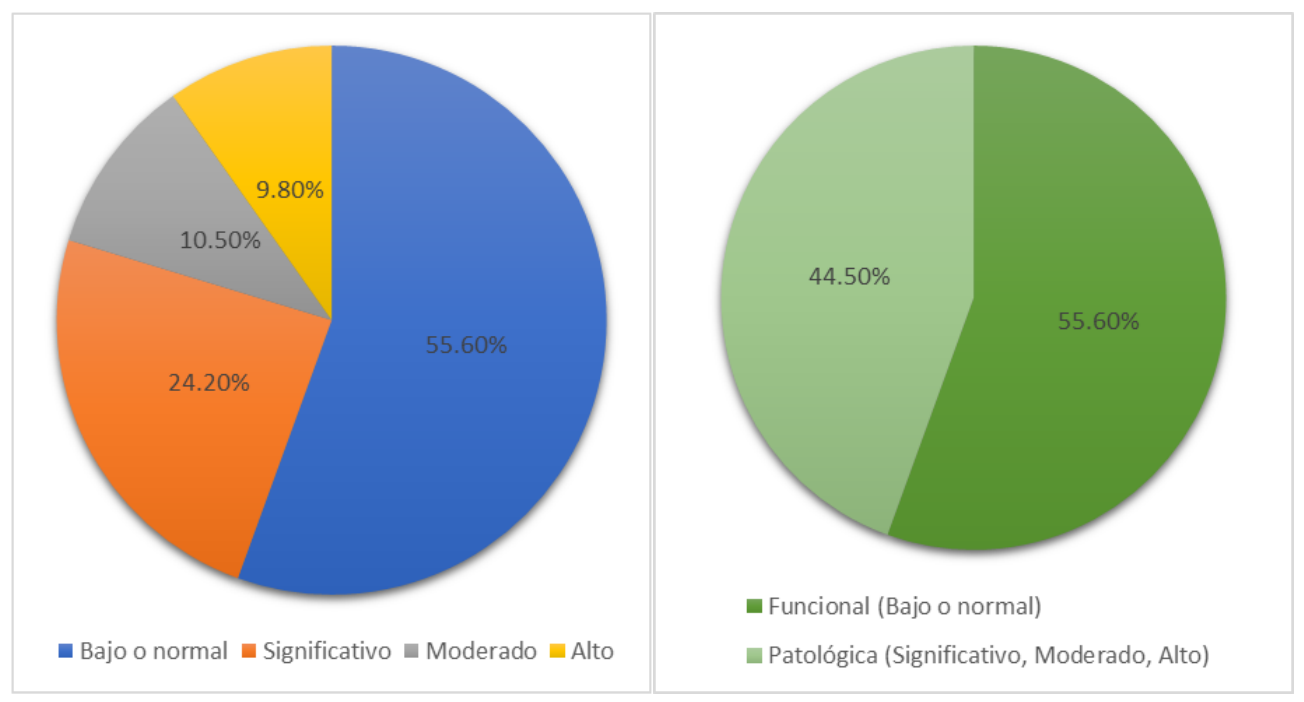


La tabla 4 indica que, el apego seguro en los participantes es más frecuente hacia la figura materna (57.8\%). El apego inseguro se refleja más hacia la figura paterna (54.2\%).

Tabla 4. Apego con la madre - Apego con el padre

\begin{tabular}{ccc}
\hline & Apego con la madre & \\
APEGO MADRE & $\mathbf{N}$ & $\%$ \\
Apego seguro & 177 & 57.8 \\
Apego inseguro & 129 & 42.2 \\
Total & 306 & 100.0 \\
& Apego con el padre & \\
APEGO PADRE & $\mathbf{N}$ & $\%$ \\
Apego seguro & 140 & 45.8 \\
Apego inseguro & 166 & 54.2 \\
Total & 306 & 100.0 \\
\hline
\end{tabular}

La tabla 5 indica que, del $55.6 \%$ (170) de los participantes que obtuvieron un nivel de dependencia emocional bajo o normal, el 37.9\% (116) reflejó un apego seguro con la madre. Mientras que, del 24.2\% (74) que obtuvo un nivel de dependencia emocional significativo, el $13.1 \%$ (40) reflejó un apego inseguro hacia la madre.

La prueba de chi-cuadrado fue realizada para determinar la relación entre el nivel de dependencia emocional y el tipo de apego hacia la madre. Esta relación fue significativa, $\mathrm{x}^{2}(3, \mathrm{~N}=306)=17.252^{\mathrm{a}}, p=.001$.

Con base a estos resultados se rechaza la hipótesis nula y se acepta que existe una relación estadísticamente significativa entre el nivel de dependencia emocional y el tipo de apego (seguro o inseguro) hacia la madre. Nota. Esta tabla contrasta la relación que existe entre la representación mental del vínculo parental temprano de la figura materna y la dependencia emocional.

Tabla 5. Dependencia emocional y apego con la madre

\begin{tabular}{lllllll}
\hline $\begin{array}{l}\text { DEPENDENCIA } \\
\text { EMOCIONAL }\end{array}$ & \multicolumn{2}{c}{ APEGO CON LA MADRE } & & \\
& Apego seguro & Apego inseguro & Total & \\
& N & $\%$ & N & \% & N & $\%$ \\
Bajo o normal & 116 & 37.9 & 54 & 17.6 & 170 & 55.6 \\
Significativo & 34 & 11.1 & 40 & 13.1 & 74 & 24.2 \\
Moderado & 13 & 4.2 & 19 & 6.2 & 32 & 10.5 \\
Alto & 14 & 4.6 & 16 & 5.2 & 30 & 9.8 \\
Total & 177 & 57.8 & 129 & 42.2 & 306 & 100.0 \\
\hline
\end{tabular}

En la tabla 6 se observa que, del 55.6\% (170) de los participantes que obtuvieron un nivel de dependencia emocional bajo o normal, el 29.4\% (90) reflejó un apego seguro hacia el padre. Mientras que, del 24.2\% de la muestra con un nivel de dependencia emocional significativo, el 17.6\% mostró un apego inseguro hacia el padre. La prueba de chi-cuadrado fue realizada para determinar la relación entre el nivel de dependencia emocional y el tipo de apego hacia el padre. Esta relación fue significativa, $\mathrm{x}^{2}(3, \mathrm{~N}=306)=14.237^{\mathrm{a}}, p=.003$.

Con base a estos resultados se rechaza la hipótesis nula y se acepta que existe una relación estadísticamente significativa entre el nivel de dependencia emocional y el tipo de apego (seguro o inseguro) hacia el padre. Nota. Esta tabla contrasta la relación que existe entre la representación mental del vínculo parental temprano de la figura paterna y la dependencia emocional. 
Jenifer Pinzón Agrazal, et al. DOI: https://doi.org/10.37387/ipc.v10i1.271

Tabla 6. Dependencia emocional y apego con el padre

\begin{tabular}{|c|c|c|c|c|c|c|}
\hline \multirow{3}{*}{$\begin{array}{l}\text { DEPENDENCIA } \\
\text { EMOCIONAL }\end{array}$} & \multicolumn{4}{|c|}{ APEGO CON EL PADRE } & \multirow{2}{*}{\multicolumn{2}{|c|}{ Total }} \\
\hline & \multicolumn{2}{|c|}{ Apego seguro } & \multicolumn{2}{|c|}{ Apego inseguro } & & \\
\hline & $\mathbf{N}$ & $\%$ & $\mathbf{N}$ & $\%$ & $\mathbf{N}$ & $\%$ \\
\hline Bajo o normal & 90 & 29.4 & 80 & 26.1 & 170 & 55.6 \\
\hline Significativo & 20 & 6.5 & 54 & 17.6 & 74 & 24.2 \\
\hline Moderado & 16 & 5.2 & 16 & 5.2 & 32 & 10.5 \\
\hline Alto & 14 & 4.6 & 16 & 5.2 & 30 & 9.8 \\
\hline Total & 140 & 45.8 & 166 & 54.2 & 306 & 100.0 \\
\hline
\end{tabular}

\section{Discusión y conclusiones}

Los resultados encontrados en este estudio muestran que existe una relación estadísticamente significativa entre el vínculo parental temprano, es decir, el tipo de apego que se tiene en la infancia hacia los padres, y la dependencia emocional en las relaciones de pareja. Estos resultados coinciden con la literatura (Barroso, 2011), la cual señala que las características, positivas o negativas del estilo de apego con nuestros padres o cuidadores en la infancia, van a determinar la calidad de nuestras relaciones afectivas en la vida adulta.

Diversos estudios han señalado que existe una relación causal entre las experiencias de un individuo con sus padres y su posterior capacidad para establecer vínculos afectivos (Bowlby, 1920).

Estévez, Chávez, Momeñe, Olave, Vázques y Juaregui (2018) señalan que, las personas emocionalmente dependientes en la vida adulta, tuvieron un apego inseguro durante la infancia. Estos resultados concuerdan con los obtenidos en nuestra investigación, que indican que el $28 \%$ de los participantes que reflejaron una vinculación afectiva patológica (nivel de dependencia emocional fuera de lo normal y no adaptativo), tenían a su vez, un apego inseguro hacia la figura paterna.

En 2010, Gomes y Bosa realizan la investigación: "Representaciones mentales del apego y la percepción de las prácticas parentales por parte de los adultos jóvenes". Los resultados obtenidos por estos investigadores coincidieron con el modelo propuesto por Bakermans - Kranenburg (2006) que dice que, las personas que han recibido un soporte seguro por parte de sus padres, han adquirido el conocimiento y tendrán acceso a este al momento de establecer vínculos con personas significativas en su vida adulta. Estos resultados se asemejan a los obtenidos en esta investigación; el 55.6\% (170) de los participantes obtuvieron un nivel de dependencia emocional bajo o normal, es decir funcional. Más de la mitad de estos participantes obtuvieron, a la vez, un apego seguro hacia la madre. Lo que refleja cómo estos patrones de apego han perdurado en el individuo y han sido puestos en práctica en sus relaciones de pareja actuales.

La relación estadísticamente significativa entre los vínculos parentales tempranos y la dependencia emocional obtenida en esta investigación, concuerda con la teoría de apego propuesta por Bowlby, que planteaba que esta relación establecida en la infancia con el cuidador principal no sólo serviría como base segura para el niño, sino que tendría un impacto en su vida adulta y futuras relaciones interpersonales (Bowlby, Mirales, 2014).

A partir de los datos obtenidos en este estudio, sería interesantes conocer en el futuro, cuáles son aquellos factores, a parte de los vínculos parentales tempranos, que pueden influir en el desarrollo de una dependencia emocional en la vida adulta.

Dentro de las limitaciones de este estudio tenemos la escogencia de la muestra; la cual no pudo darse de manera aleatoria debido a la situación de pandemia por COVID-19 que atravesaba el país. La participación fue voluntaria, lo que dificultó que la distribución por universidades fuera equitativa. La no-aleatorización de la muestra impide generalizar los resultados a la población universitaria panameña. 


\section{Agradecimientos}

A la convocatoria universitaria a fondos de investigación (CUFI) de la Universidad de Panamá, ya que, en el año 2020 este proyecto fue premiado.

\section{Referencias}

Barroso, O. (2014). El apego adulto: la relación de los estilos de apego desarrollados en la infancia en la elección y dinámicas de pareja. Revista Digital de Medicina Psicosomática y Psicoterapia, 4(1). Recuperado de https://psicociencias.org/pdf_noticias/Apego_Adulto.pdf

Blasco, J. C. (2005). Dependencia emocional. Madrid, España: Alianza Editorial.

Bowlby, J. (1920). Vínculos afectivos: formación, desarrollo y pérdida (5ª ed.). Madrid, España: Morata.

Bowlby, J., \& Mirales, G. A. (2014). Vínculos afectivos: formación, desarrollo y pérdida: Edición renovada (1.a ed.). Recuperado de https://es.scribd.com/read/282801243/Vinculos-afectivos-formacion-desarrollo-yperdida-Edicion-renovada

Bretherton, I. (1992). Los orígenes de la teoría del apego: John Bowlby y Mary Ainsworth. Psicología del desarrollo, 28 (5), 759-775. https://doi.org/10.1037/0012-1649.28.5.759

Carrillo, Sonia (1999). Marv Salter Ainsworth (1913-1999). Revista Latinoamericana de Psicología, 31 (2), 383 386. ISSN: 0120-0534. Disponible en: https://www.redalyc.org/articulo.oa?id=805/80531211

Castelló, J. (2005). Dependencia emocional: características y tratamiento. Madrid, España: Alianza Editorial.

Delgado, O. (2004). Estado actual de la teoría del apego. Revista de Psiquiatría y Psicología del Niño y del Adolescente, 4(1), 65-81. Recuperado de https://psiquiatriainfantil.org/numero4/Apego.pdf

Estévez, A., Chávez-Vera, M. D., Momeñe, J., Olave, L., Vázquez, D., \& Iruarrizaga, I. (2018). El papel de la dependencia emocional en la relación entre el apego y la conducta impulsiva. Anales de Psicología, 34(3), 438-445. https://doi.org/10.6018/analesps.34.3.313681

Gomes, V. F., \& Bosa, C. A. (2010). Representações mentais de apego e percepção de práticas parentais por jovens adultas. Psicologia: Reflexão e Crítica, 23(1), 11-18. https://doi.org/10.1590/s010279722010000100003

Gonzáles-Estela H. y Leal-Zavala R. Dependencia emocional como factor de riesgo para la violencia de pareja en mujeres del Sector Paraíso- Corregimiento Mateo Iturralde - Distrito de San Miguelito - Panamá. Rev. Tendencias en Psicología. 2014,1(2): 25-35. Recuperado de http://mail.upagu.edu.pe/files_ojs/journals/22/articles/332/submission/proof/332-97-1162-1-1020161207.pdf

Moneta, M. E. (2014). apego y pérdida: redescubriendo a John Bowlby. Chil Pediatr, 265.

Robles Estrada, E., Oudhof van Barneveld, H., \& Mercado Maya, A. (2016). Validity and reliability of the Parental Bonding Instrument (PBI) in a sample of Mexican males. Psicogente, 19(35), 14-24. https://doi.org/10.17081/psico.19.35.1205

Rodríguez, I. (2013). La dependencia emocional en las relaciones interpersonales. Revista electrónica de investigación Docencia Creativa, 2, 143-148. Recuperado de https://digibug.ugr.es/bitstream/handle/10481/27754/ReiDoCrea-Vol.2-Art.19Rodriguez.pdf?sequence $=1 \&$ isAllowed $=$ yamp/ 